\title{
10. RadiologieKongressRuhr: 9. - 11.11.2017 in Bochum
}

\section{Jahre RKR - Feiern Sie mit uns!}

Mit 700 Teilnehmern im Jahr 2008 fing alles an. Mittlerweile hat der RadiologieKongressRuhr rund 1400 Teilnehmer und über 60 Aussteller, die sich jedes Jahr in Bochum zum größten deutschen Regionalkongress der medizinischen Bildgebung treffen.

2017 begehen wir das 10-jährige Kongressjubiläum. Das macht uns zugleich stolz und dankbar. Stolz sind wir darauf, dass die Geschichte des Kongresses eine einzigartige Erfolgsgeschichte ist. Dankbar sind wir all den Menschen gegenüber, die dies durch ihren unermüdlichen Einsatz ermöglicht haben.

Anlässlich des 10-jährigen Jubiläums findet der „Empfang der Stadt Bochum“ am ersten Kongressabend an einem ganz besonderen Ort statt. Mit der Jahrhunderthalle Bochum steht uns hierfür einer der markantesten Eckpfeiler einer Industriekultur zur Verfügung, wie sie typisch ist für das Ruhrgebiet im 21. Jahrhundert. Hier, in der ehemaligen Gaskraftzentrale des Bochumer Vereins für Gussstahlfabrikation, wollen wir mit Ihnen zusammen das Jubiläum feiern. Freuen Sie sich auf einen ganz besonderen Ort und ein umfangreiches kulturelles
Begleitprogramm, das eigens hierfür vorbereitet wird.

Das 10-jährige Jubiläum des RKR findet seinen Niederschlag auch im Kongressprogramm. Im Rahmen einer Highlight-Session mit internationaler Strahlkraft möchten wir Ihnen einen spannenden Blick auf die Welt der Radiologie und darüber hinaus bieten. Im Hauptprogramm erwarten Sie außerdem wieder hochkarätige Vorträge von renommierten Referentinnen und Referenten zu aktuellen Themen der Radiologie. Auch MTRA, MTRA-SchülerInnen und Medizinstudierende werden mit eigens für sie konzipierten Programmsessions wieder auf ihre Kosten kommen.

Der RadiologieKongressRuhr steht auch in diesem Jahr nicht nur für qualifizierte Fortbildung, sondern auch für Dialog und Austausch. Wir hoffen, Sie machen davon in den Kongresspausen und während des Rahmenprogramms reichlich $\mathrm{Ge}$ brauch.
Alle Informationen zum Programm und zur Anmeldung finden Sie voraussichtlich ab Mitte Juni auf der Homepage www.radiologiekongressruhr.de.

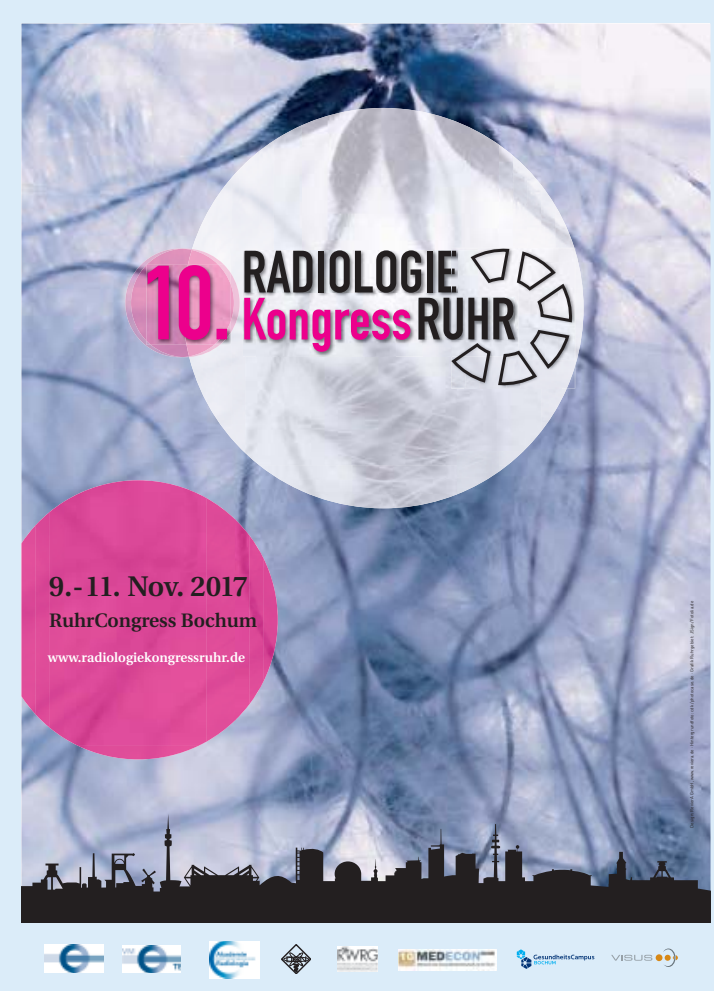

